\title{
The CAMP Lab \\ Computer Aided Medical Procedures and Augmented Reality
}

\author{
Stefanie Demirci, Pascal Fallavollita, Abouzar Eslami, \\ Diana Mateus, Tobias Lasser, Slobodan Ilic and Nassir Navab \\ Computer Aided Medical Procedures \& Augmented Reality, \\ Technical University of Munich, Munich, Germany \\ Email: see http://campar.in.tum.de/Chair/PeopleList
}

\begin{abstract}
The CAMP lab is integrated within the Department of Informatics at Technical University of Munich and is considered one of the leading groups concerned with medical augmented reality, computer assisted interventions, as well as non-medical related computer vision. In this short paper, we give an outline of the history of the lab and present a summary of some of our past and current activities relevant to augmented and virtual reality in computer assisted interventions and surgeries. References to published work in major journals and conferences allow the reader to get access to more detailed information on each subject. It was not possible to cover all aspects of our research within this paper, but we hope to provide an overview on some of these within this short paper. The readers are also invited to visit our web-site at http://campar.in.tum.de to get more information on aspects of our work. Applications for PhD and PostDoc positions can be made through the form at http://campar.in.tum.de/Chair/CampApplications.
\end{abstract}

Keywords: Computer assisted intervention/surgery; medical augmented/virtual reality

\section{INTRODUCTION}

The Chair for Computer Aided Medical Procedures \& Augmented Reality is integrated within the Department of Informatics at Technical University of Munich and is considered one of the leading groups concerned with medical augmented reality, computer assisted interventions, as well as non-medical related computer vision. It was founded in 2003 by the Technical University of Munich and Prof. Dr. Nassir Navab as first approach towards cooperation between the research fields medicine and computer science on medical imaging. In order to further realize the propagated interdisciplinarity of research, lab spaces at main Munich medical centers were established soon after the foundation.

The NARVIS lab (http://campar.in.tum.de/Chair/NarvisLab) is our research laboratory at the trauma surgery department of Munich's Ludwig-Maximilian-Universitie's medical center providing an interdisciplinary platform for our research activities on medical augmented reality (Fig. 2(a)). Since 2004, a strong collaboration between surgeons and computer scientists has been established and resulted in a large number of international publications and various research projects. Main topic of projects running at NARVIS lab addresses medical applications for Augmented Reality Technologies.

The IFL (http://campar.in.tum.de/Chair/IFL) is a central research laboratory open for joint projects of the medical and non-medical groups of the Technische Universitt Mnchen (Fig.

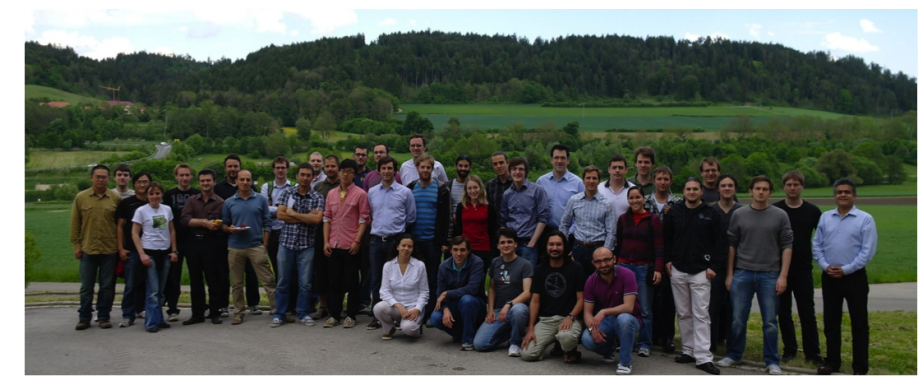

Fig. 1. The CAMP team

2(b)). Its goal is consequently to encourage interdisciplinary work and discussion between Applied Sciences, Engineering and Medicine in close contact with physicians in order to generate new lines of applied research in Medical Technology and Computer- and Robot-Aided Medical Procedures.

Within a quite short amount of time, CAMP has become one of the leading groups concerned with medical augmented reality, computer assisted interventions, as well as non-medical related computer vision. Scientists affiliated with CAMP have published in top-rank international journals and have presented their results at highly selective conferences. Since 2004, our publication list counts more than 67 papers presented at the International Conference on Medical Image Computing and Computer Assisted Interventions (MICCAI) with annual acceptance rates of about $30 \%$, and 17 presentations at the IEEE Conference on Computer Vision and Pattern Recognition (CVPR) and the International Conference on Computer Vision (ICCV) with acceptance rates of less than $30 \%$. Likewise, 16 full-length research papers have been published in the IEEE Transactions on Medical Imaging (impact factor: 3.545) and Medical Image Analysis (impact factor: 4.248).

\section{Project Overview}

The CAMP group is divided into 7 teams covering the research topics medical augmented reality and computer assisted interventions, angiographic and cardiac image processing, machine learning for medical image computing, interventional imaging, and non-medical related computer vision lead by our postdoctoral researchers. Currently, about $35 \mathrm{PhD}$ students are affiliated with the CAMP group; most of these are involved in clinic-industry-academia research projects. 


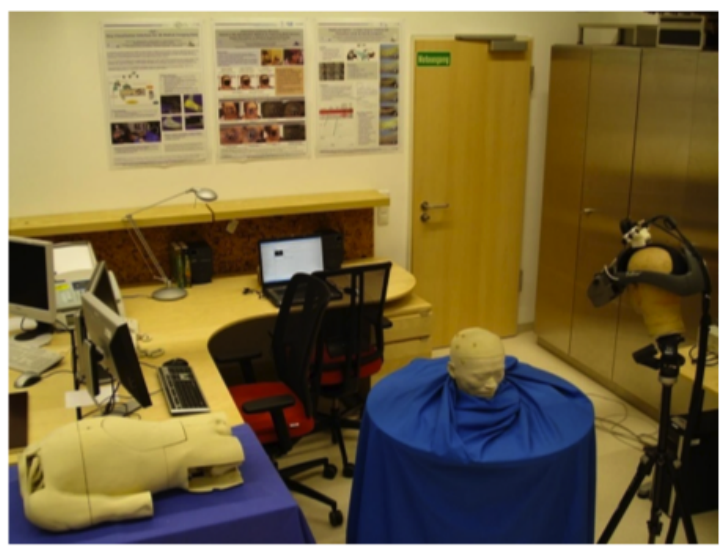

(a)

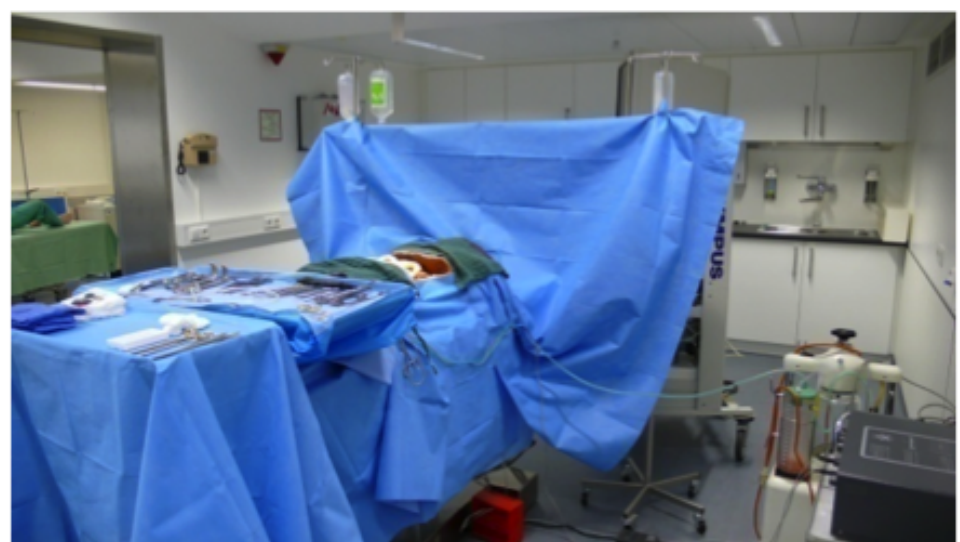

(b)

Fig. 2. CAMP labs: (a) NARVIS lab at LMU medical center, (b) IFL lab at TUM medical center

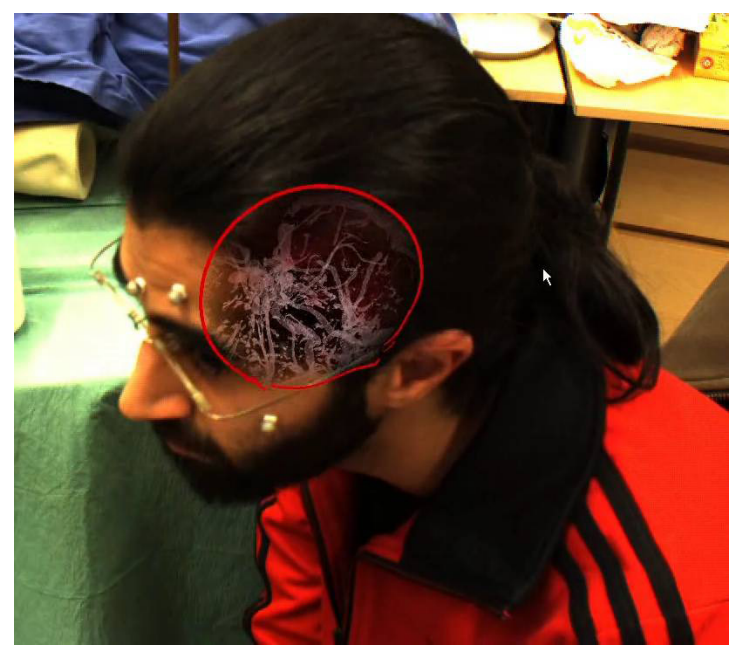

Fig. 3. Medical Augmented Reality in Abdominal (left) and Brain (right) Applications

Our objective is to create strong partnerships between clinicians, scientists and industry to define the path towards revolutionizing the design and development of physiological sensing and imaging devices in order for them to be based on and to contribute to the patient's physiological models as well as complex modelling of diagnostic and therapeutic procedures.

We believe in a strong synergy between computer vision and computer assisted inteventions. By focusing our active research in both fields, we are able to directly benefit from and transfer new results.

\section{A. Medical Augmented Reality}

Medical augmented reality (AR) has been an active subject of research in the last two decades. In the beginning, the focus was mainly on technical issues like tracking, calibration and display devices. These technologies matured in the last years and recent research focuses more on topics like usability for specific applications, user acceptance and perceptual issues.
We have presented different methods to improve usability of medical AR systems, especially for systems using a headmounted display (HMD). The virtual mirror [1] is a new interaction paradigm that allows a surgeon to take an additional viewpoint on critical structures. The user can freely move the virtual mirror inside the patient to obtain a second view of important structures, while preserving the necessary alignment between real and virtual. Regarding issues related to depth perception, when simply augmenting virtual information onto the human body, the user does not have a proper depth perception as this visualization suggest that the virtual object is in front of real objects. Contextual in-situ visualization [2], [3] significantly improves the depth perception of surgeons by superimposing augmented objects as if the user would see them through a kind of window into the body. Furthermore we have shown that missing out-of-focus blur reduces the viewing comfort in AR system and have presented a first system that can add artificial out-of-focus blur [4].

Based on this basic research we have been able to develop AR systems that are more intuitive and less stressful to use, providing additional information than traditional visualization methods. We have presented a HMD-based AR system for trajectory planning in neurosurgery [5], a very delicate task as no important structures must be injured. AR can help by providing intuitive visualization during the planning. Also for training we see a great potential of AR and have developed an AR system to teach the use of ultrasound (US) [6]. Learning how to correctly use US is very difficult as it is user dependent and US images are hard to interpret. The AR system allows seeing the US slice in-situ and provides a review environment for trainees where their own performance can be compare visually with the performance of an expert.

\section{B. $C A M C$}

The Camera Augmented Mobile C-arm (CamC) system extends a standard mobile $\mathrm{C}$-arm by a video camera and mirror construction [7], [8]. Thanks to the mirror construction and a one-time calibration of the CamC system, the acquired fluoroscopy images are co-registered with the video 


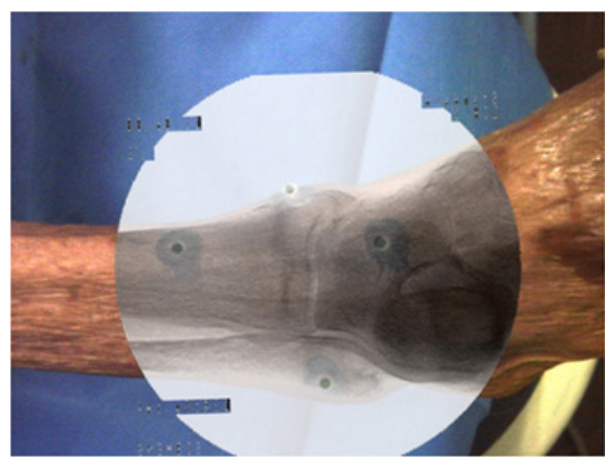

Fig. 4. CamC imaging provides significant benefits for orthopaedic and trauma surgery.

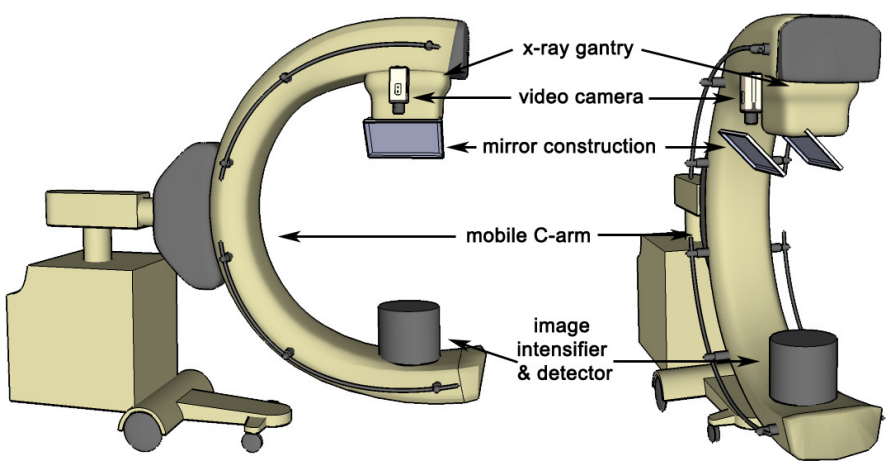

(b) images without any further calibration or registration during the intervention, providing a geometrically correct overlay. The design and imaging concept of CamC are visualized in Fig. 4(b). This allows the CamC system to provide a real-time intra-operative visualization of patient skin surface together with underlying bone structures, i.e. an X-ray and video image overlay. A workflow based method has been applied to evaluate the clinical impact of the CamC system by comparing its performance with a conventional system, i.e. standard mobile C-arm [9]. Interlocking of intramedullary nails on animal cadaver is chosen as a simulated clinical model for the evaluation study. Experimental results show that it takes significantly less radiation exposure whereas operation time for the whole interlocking procedure and quality of the drilling result are similar, using the CamC system compared to using the standard mobile $\mathrm{C}$-arm. The $\mathrm{X}$-ray and video image overlay of the CamC system enables many novel solutions for advanced $\mathrm{C}$-arm $\mathrm{X}$-ray imaging and surgical navigation. The problem of positioning mobile $\mathrm{C}$-arms repositioning during surgical procedures currently requires time, skill and additional radiation. A visual servoing based method proposed by Navab et al. [10] uses a CamC system to speed up the procedure, simplify its execution and reduce the necessary radiation for $\mathrm{C}$ arm positioning. The CamC system provides an accurate positioning and guidance of instruments in 2D. However, no depth control was possible. As an extension to the CamC system, Traub et al. [11] presented a multi-view opto-xray imaging system that is also capable of depth control during trauma surgery and orthopedic procedures using only one additional $\mathrm{X}$-ray image and a second video camera that is rigidly attached to the $\mathrm{C}$-arm. Multiple $\mathrm{C}$-arm X-ray images are acquired in order to help clinicians evaluate long bone geometry. However, impromptu and accurate intra-operative evaluation based on separated individual images remains challenging. It would be desirable to present clinicians with an X-ray panorama by stitching the individual X-ray images. Wang et al. developed a method [12]-[14] to create parallax-free panoramic X-ray images without the requirement of overlapping $\mathrm{X}$-ray regions using the CamC system.

\section{C. $4 D$ Reconstruction}

Real-time 3D-reconstruction has sparked the interest of computer vision researchers for at least the last two decades. However, using a real-time $3 \mathrm{D}$ reconstruction system for interventional applications has up to now been a little considered subject. At CAMP we developed a reconstruction system targeted at interventional environments to show that bringing such a system into a clinical environment opens the door to many new and innovative applications.

Our system is based on a total of 16 synchronized ceilingmounted cameras observing the scene. Using the images acquired by the cameras we perform a 3D-reconstruction. We designed the system with two goals in mind: real-time performance and ease of use in interventional environments. To achieve real-time performance we distributed the computations over multiple PCs making use of modern multicore architectures and GPU processing [15]. For making the system usable in an interventional setting we adopted an efficient and easy-to-use calibration procedure and a robust background subtraction algorithm which provides the input to the reconstruction algorithm. The reconstruction is performed by either fully computing the visual hull of the scene on the GPU in real-time [15] or by updating the visual hull over time [16].

Based on our system we implemented three interventional applications. In a first application we used the system to automatically detect collisions between automated medical devices such as $\mathrm{C}$-arms and other objects inside the interventional room [17] (see Fig. 5). In a second application we used the 3D-reconstruction of the scene to model a physician's radiation exposure. To this end we track the physician's reconstruction and accumulate the radiation he receives from the X-ray source over time [18]. The result is a color-coded map superimposed on the physician's reconstruction showing the areas of high radiation exposure. Finally, we also used the system to learn and recover the work flow of a surgical procedure [19].

The promising experimental results obtained with these applications show the possible impact of our work for the medical community and illustrate the clinical value of multicamera systems in the development of intelligent, integrated 

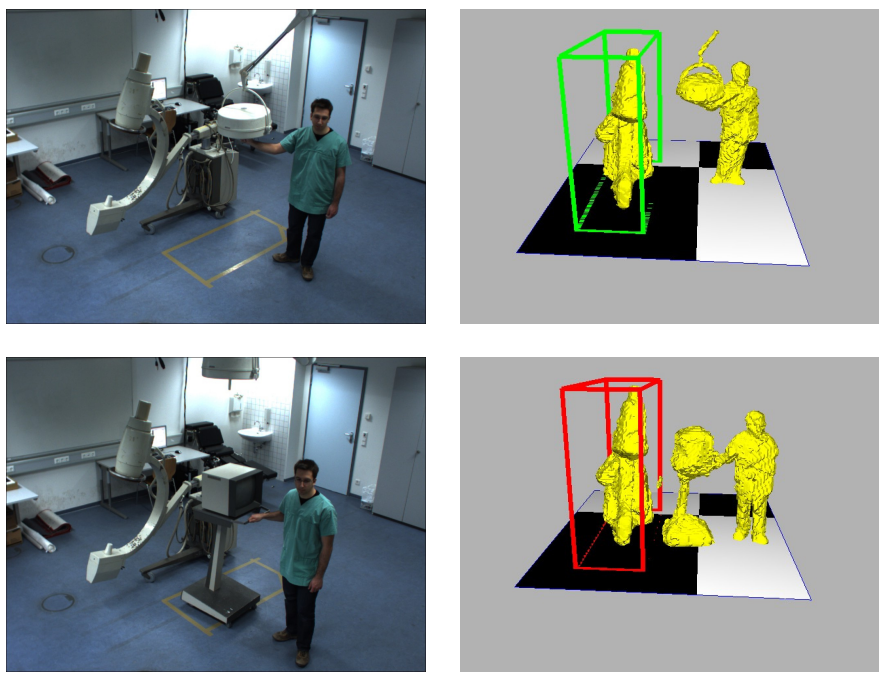

Fig. 5. Collision avoidance with medical devices. The red bounding box indicates a danger of collision. [17]

interventional suites.

In addition to the work directly related to interventional applications we also developed a method for creating highquality reconstructions from image-collections using an iterative graph-cut approach [20].

\section{CONCLUSION}

The CAMP lab is integrated within the Department of Informatics at Technical University of Munich and is considered one of the leading groups concerned with medical augmented reality, computer assisted interventions, as well as non-medical related computer vision. Its success is mostly grounded in the interdisciplinary character that is further highlighted by various sublabs located directly at Munich's major medical centers. Through the very close collaboration with the trauma surgery department, NARVIS lab is concerned with medical augmented reality whereas researchers at IFL focus on interventional medical imaging and computer assisted interventions. We plan to establish more such hospital labs and are in current negotiations with the German Heart Center as we more and more extend our research on cardiovascular related topics within computer aided diagnosis and surgery. We are also very happy to announce that CAMP is also affiliated with the prestigeous Helmholtz center via a joint research group that was created in spring 2011.

The readers are also invited to visit our web-site at http://campar.in.tum.de to get more information on aspects of our work. Applications for $\mathrm{PhD}$ and PostDoc positions can be made through the form at http://campar.in.tum.de/Chair/CampApplications.

\section{REFERENCES}

[1] C. Bichlmeier, S. Heining, M. Feuerstein, and N. Navab, "The virtual mirror: A new interaction paradigm for augmented reality environments," IEEE Transaction on Medical Imaging, vol. 28, no. 9, pp. 1498 $1510,2009$.
[2] C. Bichlmeier, S. Holdstock, S. Heining, S. Weidert, E. Euler, O. Kutter, and N. Navab, "Contextual in-situ visualization for port placement in keyhole surgery: Evaluation of three target applications by two surgeons and eighteen medical trainees," in Proc. IEEE and ACM Int. Symp. Mixed and Augmented Reality (ISMAR), 2009, pp. 173-174.

[3] C. Bichlmeier, E. Euler, T. Blum, and N. Navab, "Evaluation of the virtual mirror as a navigational aid for augmented reality driven minimally invasive procedures," in Proc. IEEE and ACM Int. Symp. Mixed and Augmented Reality (ISMAR), 2010, pp. 91-97.

[4] T. Blum, M. Wieczorek, A. Aichert, R. Tibrewal, and N. Navab, "The effect of out-of-focus blur on visual discomfort when using stereo displays," in Proc. IEEE and ACM Int. Symp. Mixed and Augmented Reality (ISMAR), 2010, pp. 13-17.

[5] R. R. Shamir, M. Horn, T. Blum, J. Mehrkens, Y. Shoshan, L. Joskowicz, and N. Navab, "Trajectory planning with augmented reality for improved risk assesment in image-guided keyhole neurosurgery," in Proc. IEEE Int. Symp. Biomedical Imaging: From Nano to Macro (ISBI), 2011, pp. 1873-1876.

[6] T. Blum, S. Heining, O. Kutter, and N. Navab, "Advanced training methods using an augmented reality ultrasound simulator," in Proc. IEEE and ACM Int. Symp. Mixed and Augmented Reality (ISMAR), 2009, pp. $177-178$.

[7] N. Navab, A. Bani-Hashemi, and M. Mitschke, "Merging visible and invisible: two camera-augmented mobile c-arm (camc) applications," in Proc. IEEE and ACM Int Workshop Augmented Reality (IWAR), 1999, pp. 134-141.

[8] N. Navab, S. Heining, and J. Traub, "Camera augmented mobile carm (camc): Calibration, accuracy study and clinical applications," IEEE Transaction on Medical Imaging, vol. 29, pp. 1412-1423, 2010.

[9] L. Wang, J. Landes, S. Weidert, T. Blum, A. von der Heide, E. Euler, and N. Navab, "First animal cadaver study for interlocking of intramedullary nails under camera augmented mobile c-arm a surgical workflow based preclinical evaluation," in Proc. Int. Conf. Information Processing in Computer-Assisted Interventions (IPCAI), 2010, pp. 56-66.

[10] N. Navab, S. Wiesner, S. Benhimane, E. Euler, and S. Heining, "Visual servoing for intraoperative positioning and repositioning of mobile carms," in Proc. Int. Conf. Medical Image Computing and ComputerAssisted Intervention (MICCAI), vol. 551-560, 2006.

[11] J. Traub, H. Heibel, P. Dressel, S. Heining, R. Graumann, and N. Navab, "A multi-view opto-xray imaging system: Development and first application in trauma surgery," in Proc. Int. Conf. Medical Image Computing and Computer-Assisted Intervention (MICCAI), vol. 18-25, 2007.

[12] L. Wang, J. Traub, S. Heining, S. Benhimane, R. Graumann, E. Euler, and N. Navab, "Long bone X-ray image stitching using camera augmented mobile c-arm," in Proc. Int. Conf. Medical Image Computing and Computer-Assisted Intervention (MICCAI), 2008, pp. 578-586.

[13] L. Wang, J. Traub, S. Weidert, S. Heining, E. Euler, and N. Navab, "Parallax-free long bone x-ray image stitching," in Proc. Int. Conf. Medical Image Computing and Computer-Assisted Intervention (MICCAI), 2009, pp. 173-180.

[14] — , "Parallax-free intra-operative x-ray image stitching," Medical Image Analysis, vol. 14, no. 5, pp. 674 - 686, 2010.

[15] A. Ladikos, S. Benhimane, and N. Navab, "Efficient visual hull computation for real-time 3D reconstruction using cuda," in Proc. IEEE CVPR Workshop Visual Computer Vision on GPUs (CVGPU), 2008, pp. 1-8.

[16] A. Bigdelou, A. Ladikos, and N. Navab, "Incremental visual hull reconstruction," in Proc. British Machine Vision Conf. (BMVC), 2009.

[17] A. Ladikos, S. Benhimane, and N. Navab, "Real-time 3D reconstruction for collision avoidance in interventional environments," in Proc. Int. Conf. Medical Image Computing and Computer-Assisted Intervention (MICCAI), 2008, pp. 526-534.

[18] A. Ladikos, C. Cagniart, R. Gothbi, M. Reiser, and N. Navab, "Estimating radiation exposure in interventional environments," in Proc. Int. Conf. Medical Image Computing and Computer-Assisted Intervention (MICCAI), 2010, pp. 237-244.

[19] N. Padoy, D. Mateus, D. Weinland, M.-O. Berger, and N. Navab, "Workflow monitoring based on 3D motion features," in Proc. ICCV Workshop Video-oriented Object and Event Classification, 2009, pp. 585-592.

[20] A. Ladikos, S. Benhimane, and N. Navab, "Multi-view reconstruction using narrow-band graph-cuts and surface normal optimization," in Proc. British Machine Vision Conf. (BMVC), 2008. 